\title{
Comparison of COPD health care utilization and associated costs across patients treated with LAMA+LABA fixed-dose therapies
}

Swetha R Palli, MS; Bin Xie, PhD; Benjamin Chastek, MS; Caitlin A Elliott, MS; and Lindsay G S Bengtson, PhD

\section{What is already known about this subject}

- Fixed-dose combinations (FDCs) of long-acting muscarinic antagonists (LAMAs) + long-acting beta2-agonists (LABAs) are guideline-recommended as maintenance therapy for patients with symptomatic COPD following monotherapy.

- There are multiple LAMA+ LABA FDCs currently approved in the United States, yet there is a lack of robust clinical trial and/or real-world evidence that provides head-to-head comparison of these LAMA + LABA FDCs.

\section{ABSTRACT}

BACKGROUND: There is limited clinical trial and/or real-world evidence comparing differences among currently approved fixed-dose combination (FDC) long-acting muscarinic antagonist (LAMA)/long-acting beta2-agonist (LABA) treatments.

OBJECTIVE: To compare chronic obstructive pulmonary disease (COPD)-related and allcause health care resource utilization (HCRU) and costs between COPD patients initiating tiotropium (TIO)+olodaterol (OLO) versus (a) other LAMA+ LABA FDCs and (b) umeclidinium (UMEC)+vilanterol (VI), specifically.

METHODS: In this retrospective observational study, patients initiating fixed-dose LAMA + LABA therapy (earliest fill date = index date) between January 1, 2014, and

\section{What this study adds}

- This analysis compared outcomes for patients initiating therapy with tiotropium (TIO) + olodaterol (OLO) with other FDC LAMA + LABAs and specifically umeclidinium (UMEC)+ vilanterol (VI) in an insured U.S. population.

- In a real-world setting, patients treated with $\mathrm{TIO}+\mathrm{OLO}$ had significantly better outcomes for important measures, with lower COPD-related and allcause emergency department visits/ costs, all-cause outpatient costs, and COPD-related pharmacy fills/costs and statistically similar outcomes among the other health care settings compared with those treated with other LAMA+ LABAs or UMEC + VI.

September 30, 2018, were identified using administrative claims data from the Optum Research Database. Patients were followed post-index for 1-12 months. Follow-up was censored at the earliest occurrence of index therapy discontinuation or switch, health plan disenrollment, study end date, or reaching the maximum 12-month allowed duration. Propensity score matching of $1: 2$ was used to balance differences in baseline characteristics between cohorts for each of the 2 comparisons. Annualized population averages of HCRU and costs were calculated for each cohort as [sum of visits (or costs) for all individuals during the follow-up period] : [sum of follow-up on-treatment time for all individuals] $\times 365$ days.

RESULTS: After matching, compared with patients who initiated other LAMA+LABAs

\section{Author affiliations}

Swetha R Palli, MS, Boehringer Ingelheim Pharmaceuticals, Ridgefield, CT. Bin Xie, PhD; Benjamin Chastek, MS; Caitlin A Elliott, MS; and Lindsay G S Bengtson, PhD; Optum, Eden Prairie, MN.

\section{AUTHOR CORRESPONDENCE:}

Swetha R Palli, 203.798.4460;

swetha.palli@boehringer-ingelheim.com

J Manag Care Spec Pharm 2021;27(7):810-24

Copyright (2021, Academy of Managed Care Pharmacy. All rights reserved.

or UMEC+ $\mathrm{VI}$, patients who initiated $\mathrm{TIO}+$ OLO had $14.29 \%$ and $16.95 \%$ fewer mean annualized per-patient COPD-related emergency department (ED) visits (vs. other LAMA+LABAs: 0.49 vs. $0.59, P=0.005$; vs. UMEC + VI: 0.48 vs. $0.56, P=0.026$ ) and $3.07 \%$ and $3.14 \%$ fewer mean annualized per-patient pharmacy fills (vs. other LAMA+LABAs: 12.66 vs. $13.07, P=0.016$; vs. UMEC+VI: 12.62 vs. $13.02, P=0.022$ ), leading to $17.39 \%$ and $21.47 \%$ lower mean annualized per-patient COPD-related ED costs (vs. other LAMA+LABAs: $\$ 289$ vs. $\$ 368, P=0.003 ;$ vs. UMEC + VI: $\$ 285$ vs. $\$ 345$, $P=0.027$ ) and $4.56 \%$ and $5.67 \%$ lower mean annualized per-patient pharmacy spending (vs. other LAMA+LABAs: $\$ 3,570$ vs. $\$ 3,741$, $P<0.001$; vs. UMEC + VI: $\$ 3,556$ vs. $\$ 3,770$, $P<0.001)$ in the follow-up period. Similarly, patients in the TIO+OLO cohort had $15.63 \%$ 
and $21.17 \%$ fewer mean annualized per-patient all-cause ED visits (vs. other LAMA+LABAs: 1.08 vs. 1.37, $P<0.001 ;$ vs. UMEC + Vl: 1.08 vs. 1.28, $P=0.001), 8.29 \%$ fewer mean annualized per-patient outpatient visits (vs. UMEC+Vl: 13.28 vs. $14.48, P=0.031), 3.41 \%$ fewer mean annualized per-patient pharmacy fills (vs. other LAMA+LABAs: 56.92 vs. $58.93, P=0.028), 19.48 \%$ and $22.28 \%$ lower mean annualized perpatient all-cause ED costs (vs. other LAMA+LABAs: $\$ 755$ vs. \$971, $P<0.001$; vs. UMEC+VI: $\$ 749$ vs. $\$ 930, P<0.001)$, and $10.86 \%$ lower mean annualized per-patient outpatient setting costs (Vs. UMEC+VI: $\$ 3,348$ vs. $\$ 3,756, P=0.050$ ). There were no statistically significant differences for the other outcome measures.

CONCLUSIONS: In a real-world setting, differences in HCRU and costs were observed between FDC LAMA+LABAs, with patients initiating $\mathrm{TIO}+\mathrm{OLO}$ having lower ED visits/costs, COPD-related pharmacy fills/ costs, and all-cause pharmacy use and outpatient visits/costs than those initiating other FDC LAMA+LABAs or UMEC+VI specifically. The remaining $H C R U$ and cost measures were not significantly different.

Chronic obstructive pulmonary disease (COPD) is a chronic, disabling, and progressive respiratory condition characterized by persistent airflow limitation that incurs substantial medical burden. ${ }^{1}$ In 2014-2015, 6.4\% of U.S. adults aged $\geq 40$ years reported being diagnosed with $\mathrm{COPD},{ }^{2}$ although millions more remain undiagnosed. There are a variety of treatment options and treatment and disease management algorithms for COPD. Since 2016, the Global Initiative for Chronic Obstructive Lung Disease (GOLD) recommendations have consistently recognized dual long-acting muscarinic antagonists (LAMAs)+long-acting beta2-agonists (LABAs) as the preferred maintenance therapy for symptomatic COPD patients following monotherapy. ${ }^{3}$

In their latest update ${ }^{4}$ LAMA + LABAs are also the favored treatment class for patients switching from an inappropriate $\mathrm{LABA}+$ inhaled corticosteroid (ICS), de-escalating from triple therapy (TT) $(\mathrm{LAMA}+\mathrm{LABA}+\mathrm{ICS})$, and/or not responding to an ICS-containing regimen. These best practice recommendations also include intra-class switching of the inhaler device or molecules in case of an unsatisfactory response. The newly released American Thoracic Society clinical practice guidelines also strongly recommend the use of LAMA+ LABA therapy over monotherapy in patients with COPD who complain of dyspnea or exercise intolerance, ${ }^{5}$ aligning with numerous meta-analyses that have documented dual bronchodilation's superior efficacy versus their mono-components. ${ }^{6-8}$

However, few head-to-head studies have examined the differences between the LAMA+LABA fixed-dose combinations (FDCs) currently approved in the United States: once-daily tiotropium (TIO)+olodaterol (OLO) $5 / 5 \mu \mathrm{g}$
(Stiolto Respimat) and umeclidinium (UMEC)+vilanterol (VI) 62.5/25 $\mu \mathrm{g}$ (Anoro Ellipta) and twice-daily glycopyrronium (GLY)+indacaterol (IND) 27.5/12.5 $\mu \mathrm{g}$ (Ultibro Breezhaler), glycopyrrolate+formoterol (FORM) 18/9.6 $\mu \mathrm{g}$ (Bevespi Aerosphere), and aclidinium (ACLI)+FORM 400/12 $\mu g$ (Duaklir Pressair). Calzetta et al. conducted an indirect comparison meta-analysis and found no significant differences among LAMA+LABAs. ${ }^{6}$ They also concluded that their effectiveness gradient (UMEC+VI $\equiv \mathrm{GLY}+\mathrm{IND}$ 15.6/27.5 $\mu \mathrm{g}>\mathrm{GLY}+\mathrm{IND} 50 / 110 \mu \mathrm{g}>\mathrm{TIO}+\mathrm{OLO}$ ) was a weak indicator of possible differences between LAMA+LABA FDCs since it was limited by substantial publication bias and that only direct comparisons would determine if a specific LAMA+LABA FDC was better than another. Their findings were contradicted by a recent network meta-analyses employing a novel improved bidimensional surface under the cumulative ranking curve analysis to combine efficacy and safety while ranking LAMA+LABAs: $\mathrm{TIO}+\mathrm{OLO}>\mathrm{GLY}+\mathrm{IND} 15.6 / 27.5 \mu \mathrm{g}>\mathrm{UMEC}+\mathrm{VI} \approx \mathrm{ACLI}+\mathrm{FOR}$ $\mathrm{M}>\mathrm{GLY}+\mathrm{IND} 50 / 110 \mu \mathrm{g}>\mathrm{GLY}+\mathrm{FORM} 14.4 / 9.6 \mu \mathrm{g} .{ }^{9}$

Only 2 head-to-head direct comparison studies of specific LAMA+LABAs have been reported to date. First, an open-label crossover randomized controlled trial (RCT) involved 236 symptomatic COPD patients receiving $\mathrm{UMEC}+\mathrm{VI}$ followed by TIO+OLO for 8 weeks each with an interim 3-week washout or vice versa. ${ }^{10}$ UMEC+VI was found to be noninferior and superior to $\mathrm{TIO}+\mathrm{OLO}$ in the per-protocol and intent-to-treat populations, respectively, for trough forced expiratory volume in 1 second (FEV1) change from baseline to week 8 . However, this difference (52 $\mathrm{mL}$ ) was lower than the minimal clinically important difference of $60 \mathrm{~mL}$ between active comparators (per the European Medicines Agency guidelines on clinical investigation of products for COPD treatment). ${ }^{11}$ While patients receiving $\mathrm{UMEC}+\mathrm{VI}$ had 2-fold odds of experiencing a clinically meaningful increase $(\geq 100 \mathrm{~mL})$ in trough FEV1 versus $\mathrm{TIO}+\mathrm{OLO}$, no significant differences in symptom improvement were observed between the groups. Furthermore, the decrease in rescue medication use ( -0.25 puffs/day) reported in this trial ${ }^{10}$ and in another real-world observational analysis by Moretz et al. (2019) $(<0.1 \text { puff/day })^{12}$ for UMEC+VI did not meet the clinically meaningful threshold of $\geq 3$ puffs/day necessary for treatment reevaluation. ${ }^{13}$ The same real-world study also found a slightly higher adherence for $\mathrm{UMEC}+\mathrm{VI}$ versus $\mathrm{TIO}+\mathrm{OLO}$ (proportion of days covered: 0.50 vs. 0.47, respectively; $\mathrm{P}<0.001) .{ }^{12}$ However, this study was limited by $\sim 70 \%$ of the study sample being covered under Medicare Part D (pharmacy claims only) and therefore lacking nonpharmacy data.

While the relative effectiveness studies discussed above did not provide conclusive guidance for treatment selection, 
economic impact is a critical decision-making parameter in a cost-conscious environment. The potential differential impact of various LAMA+LABAs on health care resource utilization (HCRU) is a key area of unmet need.

The current study aimed to address these gaps by comparing COPD-related and all-cause HCRU and costs between COPD patients initiating TIO+OLO versus (a) other LAMA+LABAs and (b) UMEC+VI specifically. We also evaluated these outcomes in the following subpopulations of interest: (a) GOLD: A/B, C/D; (b) age: elderly $(\geq 70$ years), nonelderly ( $<70$ years); (c) sex: male, female; and (d) insurance type: Medicare Advantage Part D (MAPD) plan, commercial.

\section{Methods}

\section{DATA SOURCE}

This retrospective noninterventional analysis used deidentified administrative medical and pharmacy claims data between January 1, 2013, and October 31, 2018 (study period) from the Optum Research Database (ORD). The ORD is geographically diverse in coverage, including enrollment information and medical and pharmacy claims for $\sim 19 \%$ of the U.S. commercially insured population and $\sim 21 \%$ of the U.S. population enrolled in MAPD plans with medical and pharmacy benefits. The study was HIPAA compliant and exempted from institutional review board review.

\section{PATIENT SELECTION AND COHORT ASSIGNMENT}

All members with $\geq 1$ medical claim with an International Classification of Diseases, Ninth or Tenth Revision, Clinical Modification (ICD-9-CM or ICD-10-CM) diagnosis code for COPD (ICD-9-CM: 491.xx-492.xx and 496.xx; ICD-10-CM: J41-J44) in any position during the study period were identified. Using National Drug Code numbers, patients initiating any LAMA+ LABA FDC were retained for the analysis.

Index date was the first outpatient pharmacy claim for a unique LAMA+LABA FDC (index treatment) during the identification period (January 1, 2014-September 30, 2018). Baseline was the 12-month period prior to and including the index date. Follow-up started the day after the index date. Patients were required to be followed for $\geq 30$ days, and patients were censored at the earliest occurrence of index therapy discontinuation (i.e., $\geq 60$-day gap following the runout of days supply between index regimen fills), switch (i.e., $\geq 30$ consecutive days for a nonindex maintenance therapy [LAMA monotherapy, free- or fixed-dose LABA+ICS, nonindex LAMA+LABA, or TT] based on the days supply on pharmacy claims), health plan disenrollment, or reaching the maximum allowed follow-up duration of 12 months (index date +365 days), or the study end date.
Patients were excluded if they were aged $<40$ years or had incomplete demographic information; $\geq 2$ distinct medical claim dates involving a diagnosis of asthma, interstitial lung disease, cystic fibrosis, and/or lung cancer during the baseline or follow-up periods; evidence of free- or fixeddose TT on the index date; or a baseline prescription for free- or fixed-dose LAMA+LABA or TT.

Using the index pharmacy claim, patients were assigned to 1 of 2 mutually exclusive cohorts: TIO + OLO or other LAMA+LABAs. Patients in the other LAMA+LABA cohort who initiated UMEC+VI were also assigned to the $\mathrm{UMEC}+\mathrm{VI}$ cohort and are therefore a subset of the larger LAMA+ LABA cohort.

\section{PATIENT CHARACTERISTICS}

Demographics were identified on the index date. Baseline clinical characteristics included Quan-Charlson comorbidity score with and without COPD ${ }^{14}$ Elixhauser score with and without COPD, ${ }^{15}$ COPD severity score ${ }^{16}$ exacerbation history (see Table 1 footnote), GOLD C/D status (i.e., $\geq 2$ moderate and/or $\geq 1$ severe exacerbation history), ${ }^{4}$ bronchodilator-naive status, oxygen therapy, provider specialty, and all-cause and COPD-related HCRU and costs.

\section{OUTCOMES}

HCRU included inpatient, emergency department (ED), physician office, hospital outpatient, and other medical (noninpatient) visits and pharmacy claims. Other medical visits are a residual category including, but not limited to, services rendered at independent laboratories and assisted living facilities and by home health providers.

Health care costs include the actual amounts paid by the insurer and the patient. Total costs were calculated and presented in categories of pharmacy and medical. The latter included the subcategories of inpatient, ED, physician office, hospital outpatient, and other medical costs. Costs were inflation-adjusted to 2018 U.S. dollars using the Consumer Price Index's medical care component. ${ }^{17}$

COPD-related HCRU and costs were analogous to the all-cause outcomes but restricted to medical claims with a COPD diagnosis in any position or pharmacy claims for COPD medications (maintenance, rescue, oral corticosteroids [OCSs], and/or antibiotic drugs listed elsewhere). Annualized population-averaged HCRU and costs outcomes were calculated for each cohort as: [sum of the outcome of interest for all individuals during the follow-up period]/ [sum of follow-up on-treatment time for all individuals] $\times 365$ days to account for variable follow-up. Wald $95 \%$ confidence limits for this ratio used the Taylor expansion to estimate the standard error. ${ }^{18}$ 


\section{TABLE 1 Baseline Patient Characteristics by Cohort Before and After Propensity Score Matching}

\begin{tabular}{|c|c|c|c|c|c|}
\hline & \multicolumn{5}{|c|}{ Before 1:2 PSM } \\
\hline & $\begin{array}{l}\text { TIO + OLO } \\
(n=4,370)\end{array}$ & $\begin{array}{c}\text { Other } \\
\text { LAMA+LABA } \\
(n=17,485)\end{array}$ & Std Diff (\%) & $\begin{array}{c}\text { UMEC + VI } \\
(n=15,669)\end{array}$ & Std Diff (\%) \\
\hline \multicolumn{6}{|l|}{ Baseline demographic and clinical characteristics } \\
\hline Age, years, mean [SD] & 71.82 [8.73] & $69.29 \quad[9.99]$ & 26.91 & $69.54 \quad[9.94]$ & 24.33 \\
\hline Male, n (\%) & $2,191(50.14)$ & $8,929(51.07)$ & -1.86 & $8,016(51.16)$ & -2.04 \\
\hline \multicolumn{6}{|l|}{ Region ${ }^{\mathrm{b}}, \mathrm{n}(\%)$} \\
\hline Northeast & $613(14.03)$ & $2,314(13.23)$ & 2.31 & $2,095(13.37)$ & 1.91 \\
\hline Midwest & $953(21.81)$ & $4,376(25.03)$ & -7.61 & $3,967(25.32)$ & -8.28 \\
\hline South & $2,477(56.68)$ & $9,504(54.36)$ & 4.68 & $8,472(54.07)$ & 5.26 \\
\hline West & 327 (7.48) & $1,291 \quad(7.38)$ & 0.38 & $1,135 \quad(7.24)$ & 0.92 \\
\hline \multicolumn{6}{|l|}{ Insurance type, n (\%) } \\
\hline Commercial & $253(5.79)$ & $4,784(27.36)$ & -60.61 & $4,117(26.27)$ & -58.14 \\
\hline Medicare & $4,117(94.21)$ & $12,701(72.64)$ & 60.61 & $11,552(73.73)$ & 58.14 \\
\hline Elixhauser comorbidity score without COPD, mean [SD] & $7.06[9.18]$ & $6.29 \quad[8.86]$ & 8.50 & $6.36[8.90]$ & 7.70 \\
\hline Baseline Charlson comorbidity score without COPD, mean [SD] & $1.61[1.84]$ & $1.47[1.78]$ & 7.94 & 1.48 [1.79] & 7.48 \\
\hline COPD severity score, mean $[S D]^{16}$ & $30.19[9.30]$ & $28.63[9.18]$ & 16.82 & $28.67 \quad[9.14]$ & 16.50 \\
\hline \multicolumn{6}{|l|}{ Baseline respiratory medication use, $\mathrm{n}(\%)$} \\
\hline Bronchodilator-naive & $2,662(60.92)$ & $11,933(68.25)$ & -15.38 & $10,691(68.23)$ & -15.34 \\
\hline Oxygen therapy & $993(22.72)$ & $3,250(18.59)$ & 10.23 & $2,944(18.79)$ & 9.71 \\
\hline Nebulized medication delivery & $1,226(28.05)$ & $4,721(27.00)$ & 2.36 & $4,146(26.46)$ & 3.58 \\
\hline \multicolumn{6}{|c|}{ Provider specialty from index pharmacy claims or baseline medical claims ${ }^{c}$} \\
\hline Pulmonology & $1,612(36.89)$ & $5,911(33.81)$ & 6.45 & $5,311(33.89)$ & 6.26 \\
\hline Primary care $^{d}$ & $1,052(24.07)$ & $4,549(26.02)$ & -4.49 & $4,117(26.27)$ & -5.07 \\
\hline Internal medicine & $923(21.12)$ & $3,869(22.13)$ & -2.44 & $3,521(22.47)$ & -3.27 \\
\hline Allied health professional & $542(12.40)$ & $2,176(12.44)$ & -0.13 & $1,883(12.02)$ & 1.18 \\
\hline Cardiology & $24 \quad(0.55)$ & $89 \quad(0.51)$ & 0.55 & $83 \quad(0.53)$ & 0.27 \\
\hline Other specialty & $215 \quad(4.92)$ & $883 \quad(5.05)$ & -0.60 & $746 \quad(4.76)$ & 0.74 \\
\hline Unknown & $2(0.05)$ & $8 \quad(0.05)$ & 0.00 & $8 \quad(0.05)$ & -0.24 \\
\hline \multicolumn{6}{|l|}{ Baseline exacerbations ${ }^{e}$} \\
\hline \multicolumn{6}{|l|}{ Severe } \\
\hline $\mathrm{n}(\%)$ & $527(12.06)$ & $1,955(11.18)$ & 2.74 & 1,754 (11.19) & 2.70 \\
\hline Count, mean [SD] & $1.21 \quad[0.54]$ & $1.18 \quad[0.51]$ & 6.48 & 1.19 [0.53] & 4.52 \\
\hline \multicolumn{6}{|l|}{ Moderate } \\
\hline $\mathrm{n}(\%)$ & 1,695 (38.79) & $6,668(38.14)$ & 1.34 & $5,947(37.95)$ & 1.71 \\
\hline Count, mean [SD] & 1.65 [1.13] & 1.57 [1.01] & 7.30 & $1.56[0.99]$ & 8.67 \\
\hline \multicolumn{6}{|l|}{ Any (moderate or severe) } \\
\hline $\mathrm{n}(\%)$ & $1,959(44.83)$ & $7,744(44.29)$ & 1.08 & $6,921(44.17)$ & 1.32 \\
\hline Count, mean [SD] & 1.75 [1.23] & $1.65[1.10]$ & 8.82 & 1.64 [1.09] & 9.82 \\
\hline Baseline GOLD C/D, n (\%) & $1,038(23.75)$ & $3,879(22.18)$ & 3.73 & 3,445 (21.99) & 4.21 \\
\hline
\end{tabular}




\section{TABLE 1 Baseline Patient Characteristics by Cohort Before and After Propensity Score Matching (continued)}

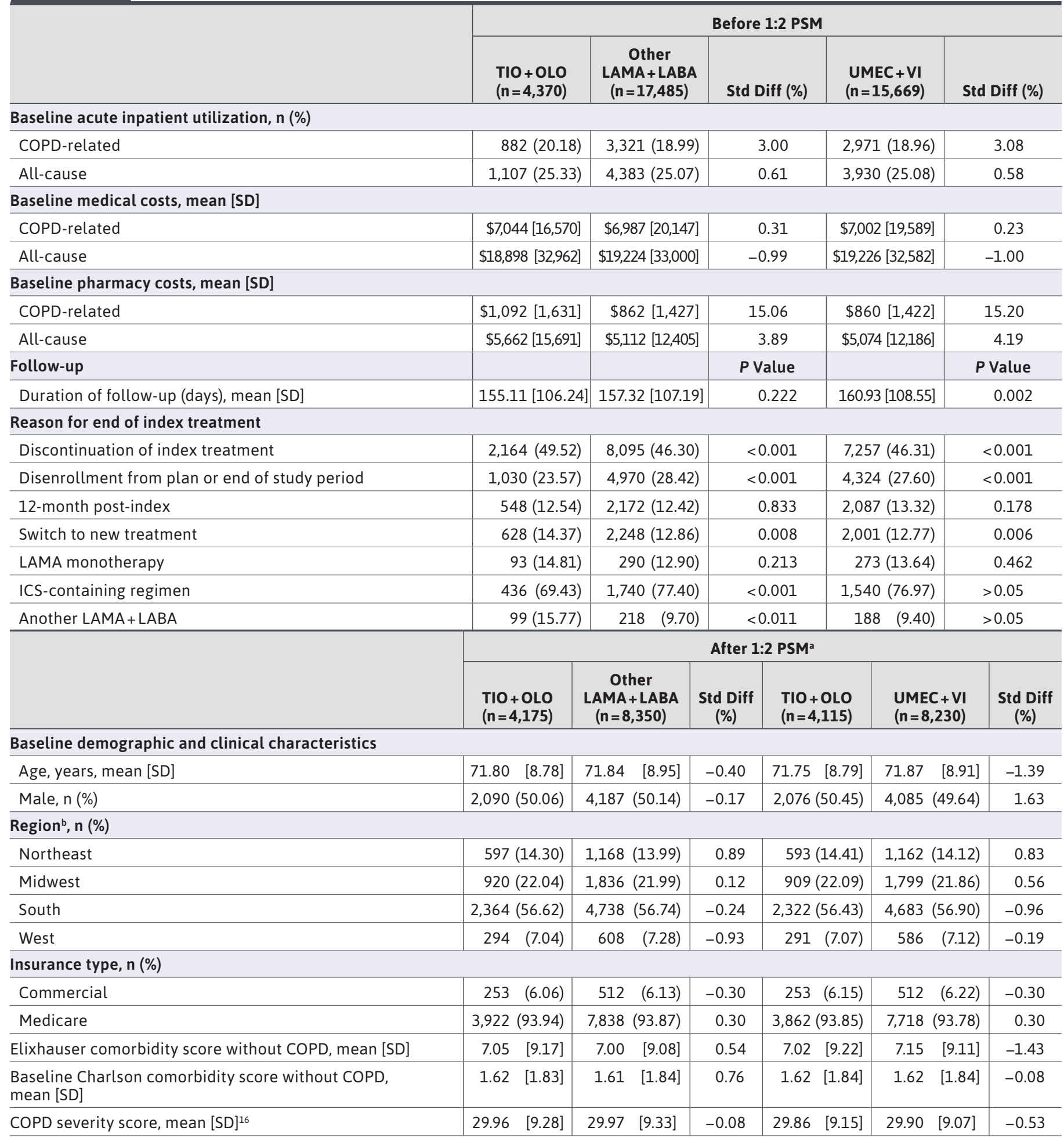




\section{TABLE 1 Baseline Patient Characteristics by Cohort Before and After Propensity Score Matching (continued)}

\begin{tabular}{|c|c|c|c|c|c|}
\hline \multicolumn{6}{|c|}{ After 1:2 PSMa } \\
\hline $\begin{array}{l}\text { TIO + OLO } \\
(n=4,175)\end{array}$ & $\begin{array}{c}\text { Other } \\
\text { LAMA+ LABA } \\
(n=8,350)\end{array}$ & $\begin{array}{c}\text { Std Diff } \\
(\%)\end{array}$ & $\begin{array}{l}\text { TIO + OLO } \\
(n=4,115)\end{array}$ & $\begin{array}{l}\text { UMEC + VI } \\
(n=8,230)\end{array}$ & $\begin{array}{l}\text { Std Diff } \\
(\%)\end{array}$ \\
\hline
\end{tabular}

Baseline respiratory medication use, $\mathrm{n}(\%)$

\begin{tabular}{|l|r|r|r|r|r|r|}
\hline Bronchodilator-naive & $2,647(63.40)$ & $5,286(63.31)$ & 0.20 & $2,632(63.96)$ & $5,234(63.60)$ & 0.76 \\
\hline Oxygen therapy & $918(21.99)$ & $1,868(22.37)$ & -0.92 & $889(21.60)$ & $1,809(21.98)$ & -0.91 \\
\hline Nebulized medication delivery & $1,165(27.90)$ & $2,372(28.41)$ & -1.12 & $1,142(27.75)$ & $2,297(27.91)$ & -0.35 \\
\hline
\end{tabular}

Provider specialty from index pharmacy claims or baseline medical claims ${ }^{c}$

\begin{tabular}{|c|c|c|c|c|c|c|}
\hline Pulmonology & $1,539(36.86)$ & $2,846(34.08)$ & 5.81 & $1,511(36.72)$ & $2,835(34.45)$ & 4.75 \\
\hline Primary care ${ }^{d}$ & $1,001(23.98)$ & 2,137 (25.59) & -3.75 & $986(23.96)$ & $2,099(25.50)$ & -3.58 \\
\hline Internal medicine & $886(21.12)$ & $1,947(23.32)$ & -5.04 & $874(21.24)$ & 1,958 (23.79) & -6.11 \\
\hline Allied health professional & $515(12.34)$ & $960(11.50)$ & 2.59 & $514(12.49)$ & 901 (10.95) & 4.80 \\
\hline Cardiology & $24 \quad(0.57)$ & $(0.46)$ & 1.67 & $24(0.58)$ & $(0.50)$ & 1.16 \\
\hline Other specialty & $208 \quad(4.98)$ & $(5.03)$ & -0.22 & $204(4.96)$ & $(4.79)$ & 0.79 \\
\hline Unknown & $2(0.05)$ & $(0.02)$ & 1.26 & $0(0.05)$ & $(0.02)$ & 1.27 \\
\hline
\end{tabular}

\section{Baseline exacerbations ${ }^{e}$}

Severe

\begin{tabular}{|c|c|c|c|c|c|c|}
\hline $\mathrm{n}(\%)$ & $510(12.22)$ & $1,025(12.28)$ & -0.18 & $488(11.86)$ & $991(12.04)$ & -0.56 \\
\hline Count, mean [SD] & $1.21[0.55]$ & {$[0.51]$} & 4.82 & $1.21[0.55]$ & {$[0.51]$} & 4.05 \\
\hline \multicolumn{7}{|l|}{ Moderate } \\
\hline $\mathrm{n}(\%)$ & $1,619(38.78)$ & 3,197 (38.29) & 1.01 & $1,590(38.64)$ & $3,171(38.53)$ & 0.22 \\
\hline Count, mean [SD] & $1.63[1.12]$ & $1.63[1.05]$ & 0.40 & $1.63[1.12]$ & [1.05] & 0.62 \\
\hline \multicolumn{7}{|l|}{ Any (moderate or severe) } \\
\hline $\mathrm{n}(\%)$ & $1,874(44.89)$ & $3,732(44.69)$ & 0.39 & $1,841(44.74)$ & $3,689(44.82)$ & -0.17 \\
\hline Count, mean [SD] & $1.74[1.23]$ & $1.72[1.15]$ & 1.65 & $1.73[1.22]$ & $1.71[1.14]$ & 1.17 \\
\hline Baseline GOLD C/D, n (\%) & $985(23.59)$ & $1,982(23.74)$ & -0.34 & $958(23.28)$ & $1,953(23.73)$ & -1.06 \\
\hline \multicolumn{7}{|l|}{ Baseline acute inpatient utilization, $\mathrm{n}(\%)$} \\
\hline COPD-related & $850(20.36)$ & $1,706(20.43)$ & -0.18 & $829(20.15)$ & $1,675(20.35)$ & -0.51 \\
\hline All-cause & $1,069(25.60)$ & $2,210(26.47)$ & -1.97 & $1,050(25.52)$ & $2,173(26.40)$ & -2.02 \\
\hline \multicolumn{7}{|l|}{ Baseline medical costs, mean [SD] } \\
\hline COPD-related & $\$ 6,995[16,832]$ & $\$ 6,722[14,822]$ & 1.73 & $\$ 6,915[16,835]$ & $\$ 6,767[14,719]$ & 0.94 \\
\hline All-cause & $\$ 18,822[32,872]$ & $\$ 18,203[25,706]$ & 2.10 & $\$ 18,837[33,135]$ & $18,557[27,393]$ & 0.92 \\
\hline \multicolumn{7}{|l|}{ Baseline pharmacy costs, mean [SD] } \\
\hline COPD-related & $\$ 1,000[1,541]$ & $\$ 979[1,497]$ & 1.34 & $\$ 968[1,490]$ & $\$ 958[1,468]$ & 0.69 \\
\hline All-cause & $\$ 5,469[14,738]$ & $\$ 5,513[12,773]$ & -0.32 & $\$ 5,433[14,085]$ & $\$ 5,406[12,556]$ & 0.20 \\
\hline Follow-up & & & $P$ Value & & & $P$ Value \\
\hline Duration of follow-up (days), mean [SD] & $152.68[104.60]$ & $159.44[108.67]$ & $<0.001$ & 152.25 [104.05] & $162.19[108.96]$ & $<0.001$ \\
\hline
\end{tabular}




\section{TABLE 1 Baseline Patient Characteristics by Cohort Before and After Propensity Score Matching (continued)}

\begin{tabular}{|c|c|c|c|c|c|c|}
\hline & \multicolumn{6}{|c|}{ After 1:2 PSM ${ }^{a}$} \\
\hline & $\begin{array}{l}\text { TIO + OLO } \\
(n=4,175)\end{array}$ & $\begin{array}{c}\text { Other } \\
\text { LAMA+ LABA } \\
(n=8,350)\end{array}$ & $\begin{array}{l}\text { Std Diff } \\
(\%)\end{array}$ & $\begin{array}{l}\text { TIO + OLO } \\
(n=4,115)\end{array}$ & $\begin{array}{l}\text { UMEC + VI } \\
(n=8,230)\end{array}$ & $\begin{array}{l}\text { Std Diff } \\
(\%)\end{array}$ \\
\hline \multicolumn{7}{|l|}{ Reason for end of index treatment } \\
\hline Discontinuation of index treatment & $2,100(50.30)$ & $3,967(47.51)$ & 0.003 & $2,083(50.62)$ & $3,896(47.34)$ & $<0.001$ \\
\hline Disenrollment from plan or end of study period & $1,011(24.22)$ & $2,120(25.39)$ & 0.138 & $994(24.16)$ & $2,087(25.36)$ & 0.132 \\
\hline 12-month post-index & $490(11.74)$ & $1,104(13.22)$ & 0.016 & $477(11.59)$ & 1,135 (13.79) & $<0.001$ \\
\hline Switch to new treatment & $574(13.75)$ & $1,159(13.88)$ & 0.839 & $561(13.63)$ & $1,112(13.51)$ & 0.850 \\
\hline LAMA monotherapy & $74(12.89)$ & $159(13.72)$ & 0.635 & $75(13.37)$ & $158(14.21)$ & 0.637 \\
\hline ICS-containing regimen & $406(70.73)$ & $888(76.62)$ & $<0.001$ & $394(70.22)$ & $839(75.45)$ & $<0.001$ \\
\hline Another LAMA+LABA & $94(16.37)$ & $112(9.66)$ & $<0.101$ & $92(16.40)$ & $116(10.34)$ & $<0.001$ \\
\hline \multirow{2}{*}{\multicolumn{7}{|c|}{$\begin{array}{l}\text { "Baseline covariates for PSM were age; age } 70 \text { or above; insurance type (commercial or MAPD plan); index year; COPD severity; ischemic heart disease; naive to } \\
\text { bronchodilators (LAMAs, LABAs) at index; oxygen therapy use; total days supply of rescue medications (albuterol, ipratropium, ipratropium/albuterol, levalbuterol, } \\
\text { metaproterenol, pirbuterol); total days supply of any monotherapy LAMA; total days supply of any other COPD medication; total days supply of any other } \\
\text { medication; ambulatory visit count for COPD; exacerbation history; sex; geographic region; Elixhauser score; depression; diabetes; hypertension; and metabolic } \\
\text { syndrome or hypercholesterolemia. } \\
\text { bPatients in the "other" US, region (e g. Puerto Rico) are not included in the study. }\end{array}$}} \\
\hline & & & & & & \\
\hline \multicolumn{7}{|c|}{$\begin{array}{l}\text { "Patients in the "other" U.S. region (e.g., Puerto Rico) are not included in the study. } \\
\text { "Measured on the pharmacy claims for the index treatment regimen on the index date or baseline medical claims among patients with an unknown provider } \\
\text { specialty from the index pharmacy claim. Patients with }>1 \text { provider specialty on the index date had a single provider assigned hierarchically in the following order: } \\
\text { pulmonology, primary care, internal medicine, allied health professional, cardiology, and other specialty. }\end{array}$} \\
\hline \multicolumn{7}{|c|}{ ¿Primary care includes family practice, general practice, and geriatrics. } \\
\hline \multicolumn{7}{|c|}{ 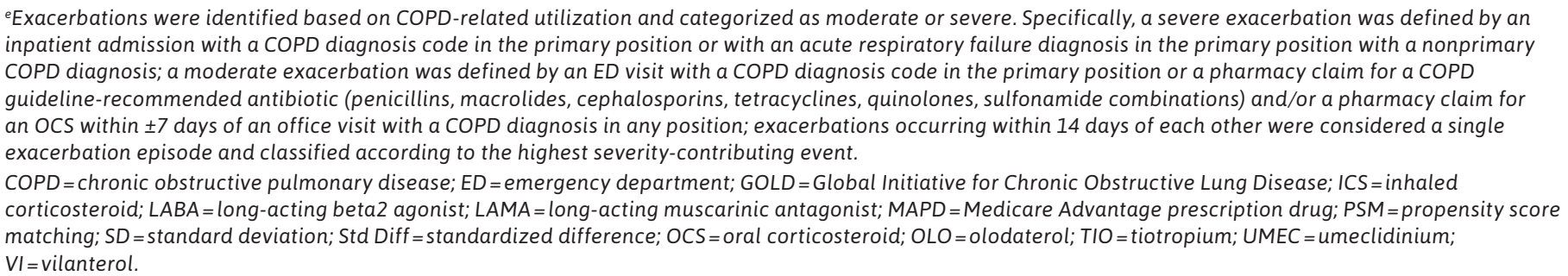 } \\
\hline
\end{tabular}

\section{STATISTICAL ANALYSIS}

Propensity score matching (PSM) was used to account for observable baseline differences between the treatment cohorts. ${ }^{19}$ Model covariates included demographics, baseline clinical characteristics, medication and exacerbation history, resource use, and medical cost quartiles. Separate analytic samples were created for each comparison, and the patients with the closest available propensity scores within each sample were matched in a 1:2 ratio. Patients were greedy matched without replacement if their propensity scores were within \pm 0.01 . The closest propensity matches were used to minimize bias in estimators. A standardized difference $<10 \%$ was considered to indicate adequate balance between study cohorts..$^{20}$

Study variables were analyzed descriptively. Outcomes were stratified by treatment cohort using 2-sample t-tests for continuous measures and Pearson chi-square tests for binary measures. Variance adjustments for the clustering due to matching were included for post-match analysis using Z-tests with robust standard errors for continuous measures and Rao-Scott tests for binary measures. A significance level $\alpha=0.05$ was used to determine statistical significance. All statistical analyses were performed using SAS version 9.4 (SAS Institute, Cary, NC).

\section{Results}

\section{PATIENT IDENTIFICATION}

Among 1,579,984 patients with a COPD diagnosis during the study period, 51,501 initiated LAMA+LABA FDC therapy during the identification period and 21,855 met all study inclusion/exclusion criteria (Figure 1). Of these, 4,370 initiated $\mathrm{TIO}+\mathrm{OLO}$. The remaining 17,485 initiated other LAMA+LABAs, with $89.6 \%$ starting UMEC+VI, 10.2\% 


\section{FIGURE 1 Patient Identification and Attrition}

$\geq 1$ claim with COPD diagnosis codes between January 1, 2013, and October 31, 2018 $\mathrm{N}=1,579,984$

$\geq 1$ unique FDC LAMA+ LABA initiation between January 1, 2014, and September 30, 2018 $\mathrm{n}=51,501$

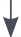

Continuous medical and pharmacy coverage for 12 months pre- and 1 month post-index date $n=29,438$

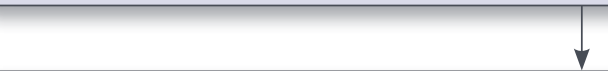

Aged $\geq 40$ years and complete demographics $\mathrm{n}=29,304$

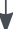

Eligible LAMA + LABA prescriptions and no index triple therapy $\mathrm{n}=28,549$

$\checkmark$

No evidence of asthma, cystic fibrosis, ILD, and/or lung cancer $n=21,855$

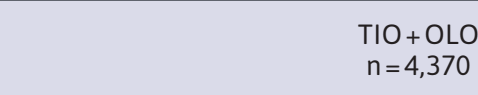

$n=4,370$

\section{Other LAMA + LABA}

$n=17,485$

UMEC + VI: 15,669; GLY + FORM: 1,790; GLY + IND: 26

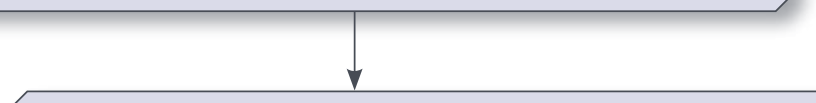

Propensity score matching

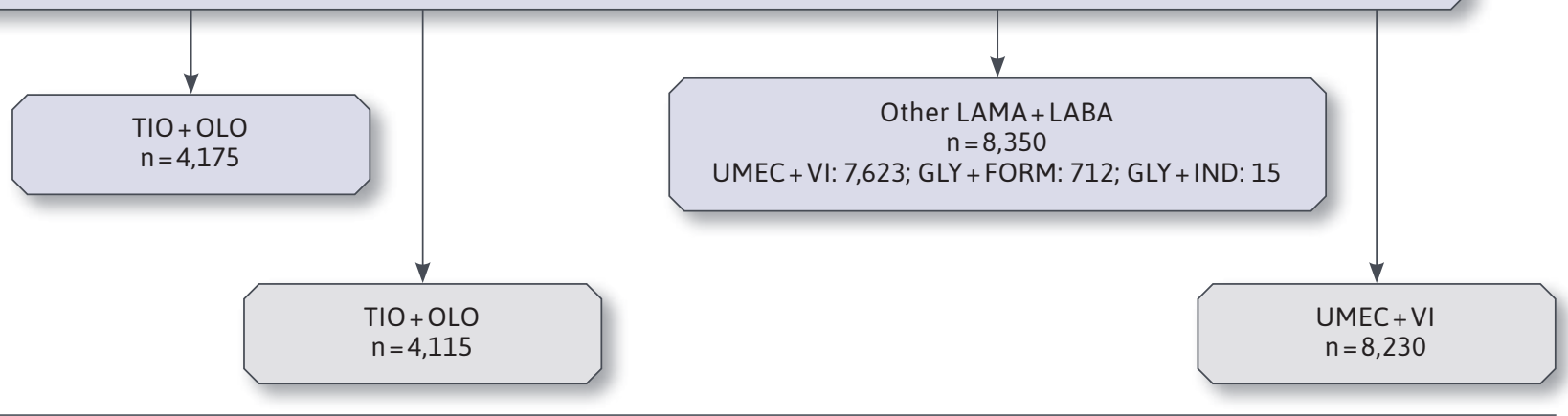

Note: Continuous enrollment criteria allow a 1-day gap in coverage.

$C O P D=$ chronic obstructive pulmonary disease; $F O R M=$ formoterol; $G L Y=$ glycopyrrolate; $I N D=$ indacaterol; $L A B A=$ long-acting beta 2 agonist; $L A M A=$ long-acting muscarinic antagonist; $\mathrm{OLO}=$ olodaterol; $\mathrm{TIO}=$ tiotropium; $\mathrm{UMEC}=$ umeclidinium; $\mathrm{VI}=$ vilanterol . 


\section{TABLE 2 COPD-Related and All-Cause HCRU: Annualized Population Averages}

\begin{tabular}{|c|c|c|c|c|}
\hline & \multicolumn{4}{|c|}{ TIO + OLO vs. Other LAMA + LABA } \\
\hline & $\begin{array}{l}\text { TIO+OLO } \\
(n=4,175)\end{array}$ & $\begin{array}{l}\text { Other LAMA + LABA } \\
\qquad(\mathrm{n}=\mathbf{8}, \mathbf{3 5 0})\end{array}$ & $\begin{array}{l}\text { Difference } \\
\text { (\%) }\end{array}$ & $\begin{array}{c}P \\
\text { Value }\end{array}$ \\
\hline \multicolumn{5}{|c|}{ COPD-related ${ }^{a}$ HCRU (annualized population average $[95 \% \mathrm{CI}]$ ) } \\
\hline Inpatient stays & {$[0.32-0.38]$} & {$[0.36-0.41]$} & $-0.03(-7.9 \%)$ & 0.141 \\
\hline Inpatient days & {$[3.52-4.78]$} & {$[3.77-4.56]$} & $-0.01(-0.2 \%)$ & 0.968 \\
\hline ED visits & {$[0.44-0.54]$} & {$[0.54-0.63]$} & $-0.10(-16.9 \%)$ & 0.005 \\
\hline Ambulatory visits & [7.14-8.03] & [7.52-8.20] & $-0.28(-3.6 \%)$ & 0.342 \\
\hline Office visits & [3.36-3.61] & [3.30-3.49] & $0.09 \quad(2.6 \%)$ & 0.245 \\
\hline Outpatient visits & [3.68-4.52] & {$[4.16-4.78]$} & $-0.37(-8.3 \%)$ & 0.164 \\
\hline Other medical visits ${ }^{\mathrm{b}, \mathrm{c}}$ & {$[3.36-3.86]$} & [3.38-3.73] & $0.05 \quad(1.4 \%)$ & 0.729 \\
\hline Pharmacy fills & $12.66[12.38-12.93]$ & $13.07[12.87-13.27]$ & $-0.41(-3.1 \%)$ & 0.016 \\
\hline \multicolumn{5}{|c|}{ All-cause HCRU (annualized population average [95\% Cl]) } \\
\hline Inpatient stays & {$[0.38-0.46]$} & {$[0.43-0.49]$} & $-0.04(-8.7 \%)$ & 0.080 \\
\hline Inpatient days & {$[4.09-5.42]$} & {$[4.37-5.22]$} & $-0.05(-1.0 \%)$ & 0.909 \\
\hline ED visits & {$[1.00-1.17]$} & [1.27-1.47] & $-0.29(-21.2 \%)$ & $<0.001$ \\
\hline Ambulatory visits & $30.11[29.01-31.21]$ & $31.00[30.20-31.79]$ & $-0.89(-2.9 \%)$ & 0.193 \\
\hline Office visits & $16.94[16.40-17.48]$ & 16.79 [16.41-17.17] & $(0.9 \%)$ & 0.659 \\
\hline Outpatient visits & 13.24 [12.37-14.11] & 14.28 [13.65-14.91] & $-1.04(-7.3 \%)$ & 0.051 \\
\hline Other medical visits ${ }^{b, c}$ & {$[8.83-9.66]$} & {$[8.96-9.62]$} & $-0.05(-0.5 \%)$ & 0.868 \\
\hline Pharmacy fills & $56.92[55.48-58.35]$ & 58.93 [57.83-60.03] & $-2.01(-3.4 \%)$ & 0.028 \\
\hline
\end{tabular}

continued on next page

starting GLY+FORM, and the remainder starting GLY+IND.

Post-match, 4,175 $\mathrm{TIO}+\mathrm{OLO}$ and 8,350 other LAMA+LABA patients were retained for the $\mathrm{TIO}+\mathrm{OLO}$ versus other $\mathrm{LAMA}+\mathrm{LABA}$ comparison; 4,115 $\mathrm{TIO}+\mathrm{OLO}$ and 8,230 UMEC+VI patients were retained for the $\mathrm{TIO}+\mathrm{OLO}$ versus $\mathrm{UMEC}+\mathrm{VI}$ comparison.

\section{PATIENT CHARACTERISTICS}

Baseline demographic and clinical characteristics before and after PSM are shown in Table 1. Prior to the match, patients in the $\mathrm{TIO}+\mathrm{OLO}$ cohort (mean age $=71.82$ years) were slightly older compared with the other LAMA+LABA (69.29 years) or UMEC+VI (69.54 years) cohorts, vs. $\$ 862$ [other LAMA+LABAs] or $\$ 860$ [UMEC+VI]). Other characteristics were similar among the cohorts. PSM successfully balanced these baseline differences, resulting in standardized differences $<10 \%$.

Patients were followed on average for 155, 157, and 161 days in the $\mathrm{TIO}+\mathrm{OLO}$, other LAMA+LABA, and UMEC+ VI cohorts, respectively. Postmatch, TIO+OLO had 2-10 fewer follow-up days. This margin is not expected to substantially influence the results. Index therapy discontinuation was the most frequent reason for follow-up termination.

\section{OUTCOMES}

COPD-Related and All-Cause HCRU. Annualized population averages of COPD-related and all-cause HCRU during follow-up are shown in Table 2. The TIO+OLO cohort had significantly fewer annualized per-patient mean COPD-related ED visits (0.49 vs. $0.59, \mathrm{P}=0.005 ; 16.95 \%$ difference) and pharmacy fills per patient (12.66 vs. $13.07, P=0.016 ; 3.14 \%$ difference) compared with those in the other LAMA+ LABA cohort. Similarly, the $\mathrm{TIO}+\mathrm{OLO}$ cohort had significantly fewer annualized mean COPD-related ED visits (0.48 vs. 0.56, $\mathrm{P}=0.026$; $14.29 \%$ difference) and pharmacy fills (12.62 vs. $13.02, \mathrm{P}=0.022 ; 3.07 \%$ difference) versus UMEC+VI. There were no statistically significant differences for the other places of service.

For all-cause HCRU, patients in the $\mathrm{TIO}+\mathrm{OLO}$ cohort had $21.17 \%$ fewer annualized mean ED visits (1.08 vs. 1.37, $\mathrm{P}<0.001$ ) and $3.41 \%$ lower annualized mean pharmacy use (56.92 vs. 58.93 , $\mathrm{P}=0.028)$ compared with those in the other LAMA+LABA cohort. Similarly, patients who initiated $\mathrm{TIO}+\mathrm{OLO}$ had $15.63 \%$ fewer annualized mean ED visits (1.08 vs. $1.28, P=0.001)$ and $8.29 \%$ fewer annualized mean outpatient visits $(13.28$ vs. $14.48, P=0.031)$ compared with those who initiated 


\section{TABLE 2}

COPD-Related and All-Cause HCRU: Annualized Population Averages (continued)

\begin{tabular}{|c|c|c|c|c|}
\hline & \multicolumn{4}{|c|}{$\mathrm{TIO}+\mathrm{OLO}$ vs. UMEC+VI } \\
\hline & $\begin{array}{l}\mathrm{TIO}+\mathrm{OLO} \\
(\mathrm{n}=4,115)\end{array}$ & $\begin{array}{l}\text { UMEC+VI } \\
(n=8,230)\end{array}$ & $\begin{array}{l}\text { Difference } \\
\text { (\%) }\end{array}$ & $\begin{array}{c}P \\
\text { Value }\end{array}$ \\
\hline \multicolumn{5}{|c|}{ COPD-related ${ }^{a}$ HCRU (annualized population average $[95 \% \mathrm{CI}]$ ) } \\
\hline Inpatient stays & {$[0.31-0.38]$} & {$[0.34-0.39]$} & $-0.02(-5.4 \%)$ & 0.347 \\
\hline Inpatient days & {$[3.51-4.81]$} & {$[3.54-4.28]$} & $0.25 \quad(6.4 \%)$ & 0.522 \\
\hline ED visits & {$[0.43-0.54]$} & {$[0.52-0.61]$} & $-0.08(-14.3 \%)$ & 0.026 \\
\hline Ambulatory visits & [7.09-7.98] & {$[7.52-8.18]$} & $-0.32(-4.1 \%)$ & 0.264 \\
\hline Office visits & [3.37-3.62] & [3.33-3.51] & $0.08 \quad(2.3 \%)$ & 0.341 \\
\hline Outpatient visits & [3.63-4.45] & [4.13-4.74] & $-0.39(-8.8 \%)$ & 0.135 \\
\hline Other medical visits ${ }^{\mathrm{b}, \mathrm{c}}$ & {$[3.28-3.77]$} & {$[3.41-3.76]$} & $-0.06(-1.7 \%)$ & 0.695 \\
\hline Pharmacy fills & $12.62[12.35-12.90]$ & $13.02[12.82-13.23]$ & $-0.40(-3.1 \%)$ & 0.022 \\
\hline \multicolumn{5}{|c|}{ All-cause HCRU (annualized population average [95\% CI]) } \\
\hline Inpatient stays & {$[0.38-0.45]$} & {$[0.42-0.47]$} & $-0.02(-4.5 \%)$ & 0.273 \\
\hline Inpatient days & {$[4.06-5.43]$} & [4.15-4.95] & $(4.2 \%)$ & 0.636 \\
\hline ED visits & {$[0.99-1.16]$} & [1.19-1.37] & $-0.20(-15.6 \%)$ & 0.001 \\
\hline Ambulatory visits & $30.23[29.12-31.34]$ & 31.12 [30.31-31.92] & $-0.89(-2.9 \%)$ & 0.207 \\
\hline Office visits & 17.02 [16.47-17.57] & $16.70[16.33-17.08]$ & $(1.9 \%)$ & 0.360 \\
\hline Outpatient visits & $13.28[12.40-14.16]$ & $14.48[13.84-15.12]$ & $-1.20(-8.3 \%)$ & 0.031 \\
\hline Other medical visits ${ }^{\mathrm{b}, \mathrm{c}}$ & {$[8.72-9.55]$} & {$[9.07-9.86]$} & $-0.33(-3.5 \%)$ & 0.273 \\
\hline Pharmacy fills & $56.80[55.36-58.23]$ & $58.54[57.46-59.63]$ & $-1.74(-3.0 \%)$ & 0.055 \\
\hline \multicolumn{5}{|c|}{$\begin{array}{l}\text { "All variables are identified during the follow-up period, excluding the index date (except the index } \\
\text { pharmacy fill). "COPD-related" defined as a medical claim with a COPD diagnosis code in any position or } \\
\text { COPD medication/guideline-recommended antibiotic. }\end{array}$} \\
\hline \multicolumn{5}{|c|}{$\begin{array}{l}\text { 'Other medical visits include services rendered at independent laboratories, assisted living facilities, and } \\
\text { urgent care clinics and by home health providers. }\end{array}$} \\
\hline \multicolumn{5}{|c|}{ 'Counted as 1 visit per provider per day. } \\
\hline \multicolumn{5}{|c|}{$\begin{array}{l}\text { Annualized population averages are calculated as the ([sum of all utilization for all individuals during } \\
\text { the follow-up period] } \div \text { [sum of follow-up on-treatment time in years ( } 365 \text { days) for all individuals] in the } \\
\text { cohort). }\end{array}$} \\
\hline \multicolumn{5}{|c|}{$\begin{array}{l}\mathrm{Cl}=\text { confidence interval; } E D=\text { emergency department; } H C R U=\text { health care resource utilization; } L A B A=\text { long- } \\
\text { acting beta2 agonist; } L A M A=\text { long-acting muscarinic antagonist; } O L O=\text { olodaterol; } T I O=\text { tiotropium; } \\
U M E C=\text { umeclidinium; } V I=\text { vilanterol. }\end{array}$} \\
\hline
\end{tabular}

$\mathrm{UMEC}+\mathrm{VI}$. There were no other statistically significant differences in other all-cause HCRU measures for both comparisons.

COPD-Related and All-Cause Costs. Annualized population averages of COPD-related and all-cause costs during follow-up are shown in Figure 2. Patients who initiated $\mathrm{TIO}+\mathrm{OLO}$ incurred significantly lower annualized per-patient mean vs. $\$ 3,770, P<0.001)$ compared with UMEC+VI. There were no other statistically significant differences with respect to COPD-related medical and total costs between the groups.

For all-cause costs, patients in the $\mathrm{TIO}+$ OLO cohort had a $22.28 \%$ difference in per-patient annualized mean ED costs ( $\$ 755$ vs. $\$ 971, P<0.001)$ versus those in the other LAMA+LABA cohort. Similarly, TIO + OLO patients incurred significantly lower perpatient mean annualized costs: $19.48 \%$ (\$749 vs. $\$ 930, \mathrm{P}<0.001$ ) and $10.86 \%$ difference $(\$ 3,348$ vs. $\$ 3,756$, $\mathrm{P}=0.050)$ in $\mathrm{ED}$ and outpatient settings, respectively, versus UMEC+VI patients. Other costs were not statistically different between $\mathrm{TIO}+\mathrm{OLO}$ and the other LAMA+LABA or UMEC+VI cohorts.

\section{SUBGROUP ANALYSIS}

In the subgroup analysis, the $\mathrm{TIO}+\mathrm{OLO}$ cohort had significantly lower annualized mean COPD-related ED visits and costs, as well as pharmacy fills and costs, in most but not all subgroups versus the other LAMA+LABA or UMEC+VI cohorts (Table 3). For example, annualized mean COPDrelated ED visits were between $3.4 \%$ (nonelderly) and 21.1\% (elderly) lower among the $\mathrm{TIO}+\mathrm{OLO}$ cohort compared with the other LAMA+LABA cohort and between 10.3\% (GOLD $\mathrm{A} / \mathrm{B}$ ) and $19.6 \%$ (elderly) lower versus UMEC+VI. Similar trends were observed for COPD-related ED costs, COPD-related and all-cause pharmacy fills and costs, and all-cause ED visits and costs within these subgroups.

ference) and fewer pharmacy fills $(\$ 3,570$ vs. $\$ 3,741, \quad \mathrm{P}<0.001 ; 4.56 \%$ difference) compared with those who initiated other LAMA+LABAs. Similarly, the TIO+OLO cohort had a significant $17.39 \%$ lower annualized mean COPD-related ED costs ( $\$ 285$ vs. $\$ 345, P=0.027$ ) and $5.67 \%$ lower annualized mean pharmacy costs $(\$ 3,556$

\section{Discussion}

In order to guide treatment decisions, understanding the impact of different treatment choices on health outcomes and associated costs in a real-world setting is important. The present 


\section{FIGURE 2 Follow-up Annualized Population Average COPD-Related and All-Cause Health Plan + Patient Costs}

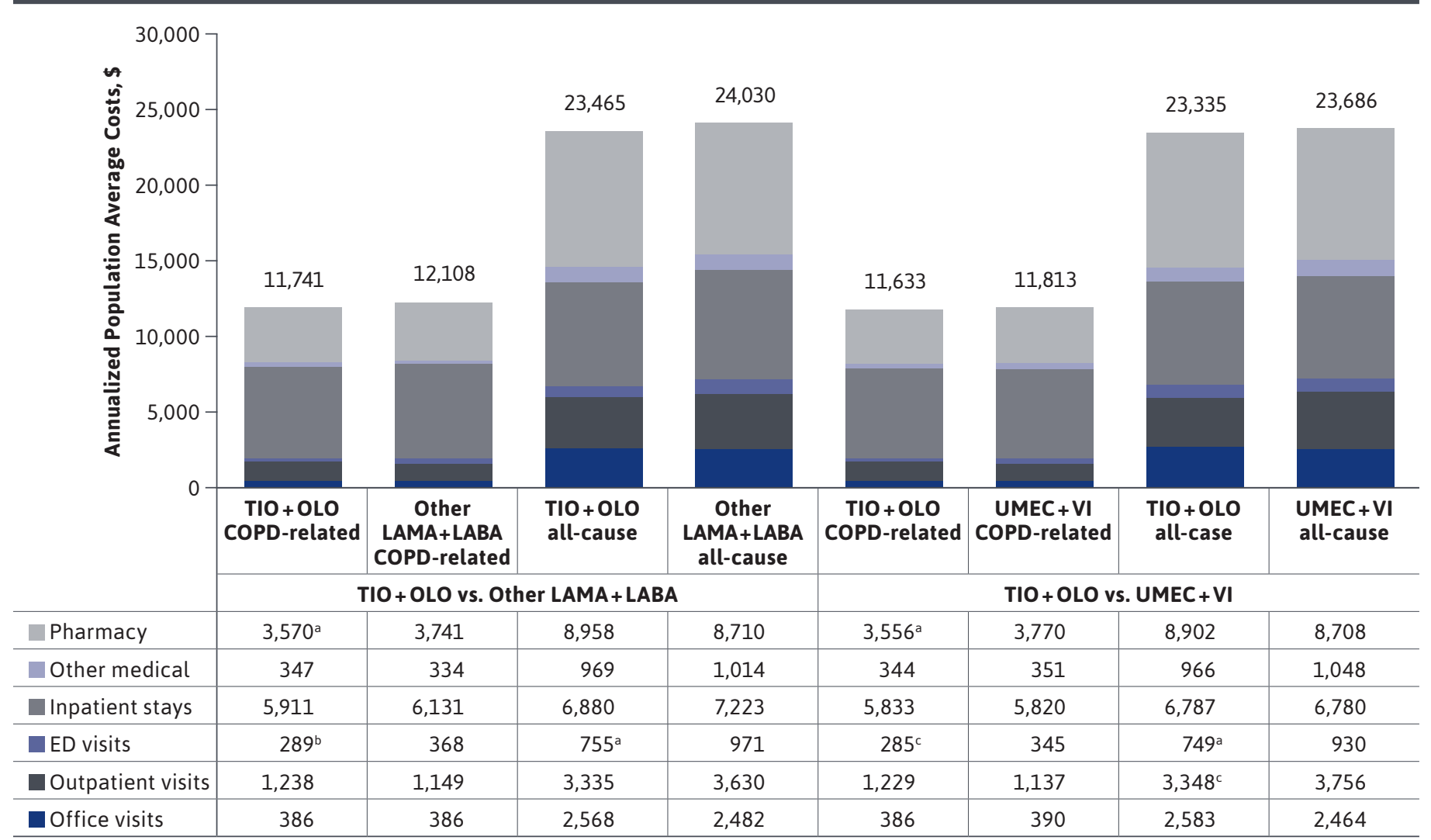

Notes: Annualized population averages are calculated as [sum of the outcome of interest for all individuals during the follow-up period] $\div$ [sum of follow-up

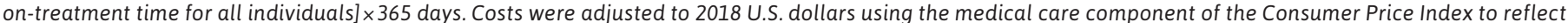
inflation between the month of the claim and the most recent complete year for which this index is available. ${ }^{19}$

ap $<0.001$.

${ }^{\mathrm{b}} \mathrm{P}<0.01$.

${ }^{c} P \leq 0.05$

$C O P D=$ chronic obstructive pulmonary disease $E D=$ emergency department; $L A B A=$ long-acting beta2 agonist; LAMA = long-acting muscarinic antagonist; $\mathrm{OLO}=$ olodaterol; $\mathrm{TIO}=$ tiotropium; UMEC = umeclidinium; $\mathrm{VI}=$ vilanterol.

study comparing COPD-related and all-cause HCRU and costs among different LAMA+LABA FDCs is also both critical and timely given that the GOLD recommendations (since 2019) suggest switching the inhaler and/or the molecule within the class before escalating a patient's current COPD treatment when symptoms persist.

Post-PSM, TIO+OLO patients had fewer annualized ED visits and lower corresponding costs, fewer annualized pharmacy fills, and lower COPD-related pharmacy spending compared with those receiving other LAMA+LABAs. Similarly, compared with patients who initiated UMEC+VI, those who initiated TIO+OLO had fewer annualized ED visits and lower costs, fewer COPD-related pharmacy fills, and lower COPD-related pharmacy costs. TIO + OLO also had lower all-cause outpatient visits, leading to lower costs for this setting.

To the extent that our results can be generalized, $\mathrm{TIO}+\mathrm{OLO}$ may be associated with fewer annualized ED visits, pharmacy fills, and (in some cases) outpatient visits and costs versus other LAMA+LABAs or UMEC+VI. It is impossible to associate the observed differences in this study to formulation differences alone, as inhalers play an integral role in COPD management, ${ }^{4,5}$ While $\mathrm{TIO}+\mathrm{OLO}$ is delivered using a slow-moving mist inhaler, both UMEC+VI and GLY+IND are dry powder inhalers, and GLY+FORM uses a pressurized metered dose inhalation 

Pharmacy HCRU Counts and Costs

\begin{tabular}{|c|c|c|c|c|c|c|c|c|c|c|c|c|}
\hline & \multicolumn{2}{|c|}{$\begin{array}{c}\text { TIO+ OLO vs. Other } \\
\text { LAMA+ LABAs }\end{array}$} & \multicolumn{2}{|c|}{$\begin{array}{l}\text { TIO + OLO vs. } \\
\text { UMEC + VI }\end{array}$} & \multicolumn{4}{|c|}{ ED Visits } & \multicolumn{4}{|c|}{ ED Costs } \\
\hline & \multirow{2}{*}{$\begin{array}{l}\mathrm{TIO+} \\
\text { OLO } \\
\text { (n) }\end{array}$} & \multirow{2}{*}{$\begin{array}{l}\text { Other } \\
\text { LAMA+ } \\
\text { LABA } \\
\text { (n) }\end{array}$} & \multirow{2}{*}{$\begin{array}{l}\text { TIO+ } \\
\text { OLO } \\
\text { (n) }\end{array}$} & \multirow{2}{*}{$\begin{array}{c}\text { UMEC+ } \\
\text { VI } \\
\text { (n) }\end{array}$} & \multicolumn{2}{|c|}{$\begin{array}{c}\text { vs. Other } \\
\text { LAMA+ LABA }\end{array}$} & \multicolumn{2}{|c|}{ vs. UMEC + VI } & \multicolumn{2}{|c|}{$\begin{array}{c}\text { vs. Other } \\
\text { LAMA+ LABA }\end{array}$} & \multicolumn{2}{|c|}{ vs. UMEC + VI } \\
\hline & & & & & $\begin{array}{l}\text { COPD- } \\
\text { related }\end{array}$ & $\begin{array}{l}\text { All- } \\
\text { cause }\end{array}$ & $\begin{array}{l}\text { COPD- } \\
\text { related }\end{array}$ & $\begin{array}{l}\text { All- } \\
\text { cause }\end{array}$ & $\begin{array}{l}\text { COPD- } \\
\text { related }\end{array}$ & $\begin{array}{l}\text { All- } \\
\text { cause }\end{array}$ & $\begin{array}{l}\text { COPD- } \\
\text { related }\end{array}$ & $\begin{array}{l}\text { All- } \\
\text { cause }\end{array}$ \\
\hline Overalla & 4,175 & 8,350 & 4,115 & 8,230 & $-16.9^{\mathrm{b}}$ & $-21.2^{\mathrm{b}}$ & $-14.3^{b}$ & $-15.6^{\mathrm{b}}$ & $-21.5^{b}$ & $-22.3^{b}$ & $-17.4^{\mathrm{b}}$ & $-19.5^{\mathrm{b}}$ \\
\hline GOLD A/B & 3,190 & 6,368 & 3,157 & 6,277 & $-19.0^{\mathrm{b}}$ & $-26.9^{c}$ & -10.3 & $-17.8^{\mathrm{d}}$ & $-20.4^{b}$ & $-25.9^{b}$ & -11.3 & $-21.1^{\mathrm{d}}$ \\
\hline GOLD C/D & 985 & 1,982 & 958 & 1,953 & -11.3 & -6.6 & -14.0 & -9.0 & -20.9 & -13.5 & -21.9 & -14.1 \\
\hline Elderly ( $\geq 70$ years) ${ }^{a}$ & 2,683 & 5,366 & 2,665 & 5,330 & $-21.1^{\mathrm{d}}$ & $-21.1^{c}$ & $-19.6^{d}$ & $-21.7^{\mathrm{d}}$ & $-24.0^{\mathrm{b}}$ & $-22.2^{b}$ & $-20.3^{b}$ & $-21.6^{d}$ \\
\hline Nonelderly ( $<70$ years) a,e & 1,427 & 2,854 & 1,394 & 2,788 & -3.4 & $-15.9^{\mathrm{b}}$ & -16.7 & $-21.8^{\mathrm{d}}$ & -16.2 & $-23.6^{\mathrm{b}}$ & $-25.8^{\mathrm{b}}$ & $-30.7^{c}$ \\
\hline MAPD $^{a}$ & 3,866 & 7,732 & 3,793 & 7,586 & $-13.6^{\mathrm{b}}$ & $-17.3^{c}$ & $-13.6^{b}$ & $-18.5^{c}$ & -14.8 & $-20.0^{c}$ & $-18.6^{\mathrm{b}}$ & $-22.7^{c}$ \\
\hline Commerciala & 225 & 450 & 228 & 456 & -14.3 & -34.6 & -19.0 & $-42.7^{b}$ & -37.4 & -32.5 & -62.6 & -61.4 \\
\hline Male $^{a}$ & 2,099 & 4,187 & 2,076 & 4,085 & -15.1 & $-19.8^{d}$ & -11.8 & $-14.9^{b}$ & $-31.4^{c}$ & $-27.8^{c}$ & $-27.9^{d}$ & $-23.1^{d}$ \\
\hline \multirow[t]{4}{*}{ Female $^{a}$} & 2,085 & 4,163 & 2,039 & 4,145 & $-17.2^{b}$ & $-21.1^{\mathrm{d}}$ & -14.8 & $-17.0^{\mathrm{b}}$ & -12.8 & $-17.2^{\mathrm{b}}$ & -7.7 & $-16.1^{\mathrm{b}}$ \\
\hline & & & & & \multicolumn{4}{|c|}{ Pharmacy Fills } & \multicolumn{4}{|c|}{ Pharmacy Costs } \\
\hline & & & & & \multicolumn{2}{|c|}{$\begin{array}{c}\text { vs. Other } \\
\text { LAMA+ LABA }\end{array}$} & \multicolumn{2}{|c|}{ vs. UMEC + VI } & \multicolumn{2}{|c|}{$\begin{array}{c}\text { vs. Other } \\
\text { LAMA + LABA }\end{array}$} & \multicolumn{2}{|c|}{ vs. UMEC + VI } \\
\hline & & & & & $\begin{array}{l}\text { COPD } \\
\text { related }\end{array}$ & $\begin{array}{l}\text { All- } \\
\text { cause }\end{array}$ & $\begin{array}{l}\text { COPD } \\
\text { related }\end{array}$ & $\begin{array}{l}\text { All- } \\
\text { cause }\end{array}$ & $\begin{array}{l}\text { COPD } \\
\text { related }\end{array}$ & $\begin{array}{c}\text { All- } \\
\text { cause }\end{array}$ & $\begin{array}{l}\text { COPD } \\
\text { related }\end{array}$ & $\begin{array}{l}\text { All- } \\
\text { cause }\end{array}$ \\
\hline Overall & 4,175 & 8,350 & 4,115 & 8,230 & $-3.1^{\mathrm{b}}$ & $-3.4^{b}$ & $-3.1^{\mathrm{b}}$ & $-3.0^{\mathrm{b}}$ & $-4.6^{b}$ & 2.8 & $-5.7^{b}$ & 2.2 \\
\hline GOLD A/B & 3,190 & 6,368 & 3,157 & 6,277 & $-3.5^{b}$ & $-3.6^{b}$ & $-3.7^{b}$ & -3.2 & $-4.3^{c}$ & -0.1 & $-5.6^{c}$ & 1.6 \\
\hline GOLD C/D & 985 & 1,982 & 958 & 1,953 & -1.0 & -2.2 & 0.1 & -1.2 & $-5.1^{\mathrm{b}}$ & 13.2 & $-5.6^{b}$ & 4.9 \\
\hline Elderly ( $\geq 70$ years) ${ }^{a}$ & 2,683 & 5,366 & 2,665 & 5,330 & $-4.3^{b}$ & $-4.4^{b}$ & -2.6 & -1.9 & $-7.3^{c}$ & -1.5 & $-7.3^{c}$ & -2.7 \\
\hline Nonelderly $(<70 \text { years })^{a, e}$ & 1,427 & 2,854 & 1,394 & 2,788 & 0.4 & -2.3 & -1.5 & -2.1 & -2.1 & 8.2 & $-5.0^{d}$ & 4.1 \\
\hline MAPD plan ${ }^{a}$ & 3,866 & 7,732 & 3,793 & 7,586 & -2.2 & $-4.6^{d}$ & -2.0 & -1.8 & $-5.6^{c}$ & 2.0 & $-6.1^{c}$ & 1.6 \\
\hline Commerciala $^{\mathrm{a}}$ & 225 & 450 & 228 & 456 & 6.0 & -2.6 & 5.0 & 1.4 & 3.6 & -10.3 & -1.8 & 12.4 \\
\hline Male $^{a}$ & 2,099 & 4,187 & 2,076 & 4,085 & $-5.4^{d}$ & $-5.1^{\mathrm{b}}$ & $-5.8^{d}$ & $-4.1^{\mathrm{b}}$ & $-4.5^{c}$ & 12.2 & $-6.0^{c}$ & 8.7 \\
\hline Female $^{a}$ & 2,085 & 4,163 & 2,039 & 4,145 & -1.0 & -2.0 & -0.3 & -1.8 & $-4.6^{c}$ & -5.9 & $-5.3^{c}$ & -4.2 \\
\hline
\end{tabular}

Note: Percent differences in HCRU and costs were calculated by dividing the absolute difference in these outcomes by the outcome value in the comparison cohorts (other LAMA+LABAs or UMEC + VI). This table presents the percent differences in ED visits and costs as well as pharmacy fills and costs for the 2 comparisons. For example, COPD-related ED visits were $16.9 \%$ lower in the TIO+OLO cohort compared to the other LAMA+LABA cohort.

aIn these stratified analyses, some patients were dropped due to hard matching, so the sample sizes of these strata may not sum to the entire sample size of the corresponding cohorts.

${ }^{b}$ Statistically significant with $P \leq 0.05$.

'Statistically significant with $P \leq 0.001$.

dStatistically significant with $P \leq 0.01$.

eWe also found COPD-related inpatient stay to be significantly lower in the nonelderly subgroup for TIO + OLO vs. UMEC + VI ( $0.27 \mathrm{vs.} 0.33 ; \mathrm{P}=0.047 ;-18.2 \%$ difference).

$G O L D=$ Global Initiative for Chronic Lung Disease; $E D=$ emergency department; $H C R U=$ health care resource utilization; $L A B A=$ long-acting beta2 agonist; $L A M A=$ long-acting muscarinic antagonist; $M A P D=$ Medicare Advantage Part D plan; $O L O=$ olodaterol; $T I O=$ tiotropium; $U M E C=u m e c l i d i n i u m ; ~ V I=v i l a n t e r o l$. 
device. Furthermore, while TIO+OLO and UMEC+VI are administered once daily, both GLY+IND and GLY+FORM are twice-daily medications that add further heterogeneity to the other LAMA+LABA cohort, making our TIO+OLO versus UMEC+VI comparison even more relevant.

Results of the present observational study differ from the comparable RCT, in which UMEC+VI was found to be noninferior or superior to $\mathrm{TIO}+\mathrm{OLO}$ in improving trough FEV1. ${ }^{10}$ This discrepancy could suggest that trough FEV1 may not translate directly to real-world clinical and/ or economic outcomes. In support of this idea, a recent cross-sectional study found that FEV1 was not a strong predictor of respiratory muscle strength, functional exercise capacity, or patient-reported dyspnea..$^{21}$ Furthermore, the direction of the current study results was partly echoed in findings from 2 prior studies. ${ }^{22,23}$ While the main objective of these studies was to compare TIO+OLO with TT, secondary analyses comparing any LAMA+ LABA versus TT showed that the observed margins of difference (vs. TT) were smaller for the any LAMA+LABA group than for $\mathrm{TIO}+\mathrm{OLO}$ specifically.

After hospitalization, ED represents the most expensive health care setting. Examining the Nationwide Emergency Department Sample, the largest publicly available U.S. sample of ED visits, the crude rate of COPD visits indicated a steady rise from 134.5 to 235.8 per 100,000 persons from 2006 to 2016. In 2016 alone, COPD accounted for 761,894 standalone ED visits plus an additional 544,781 visits that led to an inpatient admission. ${ }^{24}$ Further, the proportion of COPD patients using ED services increased by $65 \%$ between 2008-2009 and 2014-2015. ${ }^{4}$ Compounding the volume issues for ED, the mean wait time in this setting also increased. The average hospital ED patient in the United States waits 1.5 hours to be taken to an examination room and spends a total of 2.25 (1.33-5.12) hours before being discharged. ${ }^{25,26}$ These extended wait times contribute to ED crowding and an increasing number of patients leaving before seeing a doctor and/or against medical advice. ${ }^{27}$

While trends in health service use may be influenced by many factors, the current study results suggest that $\mathrm{TIO}+\mathrm{OLO}$ offers 1 potential avenue to reduce COPD-related ED utilization: the number needed to treat ranged between 10 (vs. other LAMA+ LABAs) and 13 (vs. UMEC+ VI) to avoid 1 COPD-related ED visit. In this era of evidence-based medicine and cost containment, these findings may be relevant to payers and health systems with a large COPD population and/or those with population management targets that include reducing avoidable ED visits. ${ }^{28}$

The current study also documented significantly lower COPD-related pharmacy fills and corresponding pharmacy costs. This included maintenance and rescue fills, as well as any outpatient OCS and/or antibiotic prescriptions for exacerbation management. Since we did not separate each drug class contribution, it was not feasible to compare our findings with those of Moretz et al. ${ }^{12}$

\section{STRENGTHS AND LIMITATIONS}

Limitations of this study are primarily a function of the study design and data source. It is challenging to establish causality between the various treatments and outcomes due to the retrospective design, and this study is unable to provide any insight into plausible pathways through which $\mathrm{TIO}+\mathrm{OLO}$ resulted in fewer ED and outpatient visits or lower pharmacy use. Administrative claims databases also present certain inherent limitations given that claims are collected for payment purposes, not research. Notably, the presence of a filled prescription claim does not indicate that medication was taken as prescribed. Moreover, claims do not capture medications filled over the counter or provided as samples by the physician or in a clinical trial. The presence of a diagnosis code on a medical claim is also not a confirmation of a disease condition due to entry errors, omissions, or codes being included as rule-out criteria. In our study, however, we believe this particular risk was mitigated by requiring both a COPD diagnosis and a COPD-only indicated treatment. Finally, seasonality might be associated with some of the outcomes but was not controlled for in this study.

To the best of our knowledge, this is the first realworld within-class comparison of all LAMA+LABA FDC therapies. There are several areas of strength, including a study population that is representative of a geographically diverse sample of the overall U.S. insured population. The study also employed a robust 1:2 PSM approach with the standardized difference threshold set at $10 \%$ for evaluation of the between-cohort balance (although $20 \%$ is an acceptable threshold). ${ }^{29}$

\section{Conclusions}

While GOLD recommendations provide treatment guidance to health care providers, real-world studies provide needed context on the HCRU and cost outcomes that stem from these treatment decisions. This study provides realworld HCRU and associated costs of various LAMA+LABA FDCs to aid in clinical decision making. In a real-world setting, differences in HCRU and costs were observed between LAMA+LABA FDCs, with TIO+OLO associated with lower ED visits/costs, COPD-related pharmacy fills/costs, and all-cause pharmacy use and outpatient visits/costs. 


\section{DISCLOSURES}

This study was sponsored by Boehringer Ingelheim Pharmaceuticals, Inc. (BIPI; Ridgefield, CT). BIPI was given the opportunity to review the manuscript for medical and scientific accuracy, as well as intellectual property considerations. Palli is an employee of BIPI. Xie, Chastek, Elliott, and Bengtson are employees of Optum, which was contracted by BIPI to conduct this study. The authors received no direct compensation related to the development of the manuscript.

Part of the results of this study were accepted and presented at the 30th European Respiratory Society (ERS) International Congress (September 7-9, 2020; virtual).

\section{ACKNOWLEDGMENTS}

The authors thank the following Optum study team members: Miriam Cisternas for research support; Lee Brekke for analytic support; Feng Cao, Yiyu Fang, and James Hartje for programming support; and Sharanya Murali for project management. Writing, editorial support, and formatting assistance was provided by Virginia Rosen of Optum.

\section{REFERENCES}

1. Ford ES, Murphy LB, Khavjou O, Giles WH, Holt JB, Croft JB. Total and state-specific medical and absenteeism costs of COPD among adults aged $\geq 18$ years in the United States for 2010 and projections through 2020. Chest. 2015;147(1):31-45.

2. Biener AI, Decker SL, Rohde F. Prevalence and treatment of chronic obstructive pulmonary disease (COPD) in the United States. JAMA. 2019;322(7):602.

3. Global Initiative for Chronic Obstructive Lung Disease. COPD diagnosis and management at-aglance desk reference. 2016. Accessed March 1, 2021. https://goldcopd.org/ copd-diagnosis-management-glancedesk-reference-2016/
4. Global Initiative for Chronic Obstructive Lung Disease. Pocket guide to COPD diagnosis, management, and prevention: a guide for healthcare professionals, 2020. Accessed March 1, 2021. https://goldcopd.org/wp-content/ uploads/2019/11/GOLD-Pocket-Guide2020-final-wms.pdf

5. Nici L, Mammen MJ, Charbek E, et al. Pharmacologic management of chronic obstructive pulmonary disease. An official American Thoracic Society clinical practice guideline. Am J Respir Crit Care Med. 2020;201(9):e56-e69.

6. Calzetta L, Rogliani P, Matera MG, Cazzola M. A systematic review with meta-analysis of dual bronchodilation with LAMA/LABA for the treatment of stable COPD. Chest. 2016;149(5):1181-96.

7. Calzetta L, Rogliani P, Ora J, Puxeddu E, Cazzola M, Matera MG. LABA/LAMA combination in COPD: a meta-analysis on the duration of treatment. Eur Respir Rev. 2017;26(143):160043.

8. Rodrigo GJ, Price D, Anzueto A, et al. LABA/LAMA combinations versus LAMA monotherapy or LABA/ICS in COPD: a systematic review and meta-analysis. Int J Chron Obstruct Pulmon Dis. 2017;12:907-22.

9. Rogliani P, Matera MG, Ritondo BL, et al. Efficacy and cardiovascular safety profile of dual bronchodilation therapy in chronic obstructive pulmonary disease: a bidimensional comparative analysis across fixed-dose combinations. Pulm Pharmacol Ther. 2019;59:101841.

10. Feldman GJ, Sousa AR, Lipson DA, et al. Comparative efficacy of once-daily umeclidinium/vilanterol and tiotropium/ olodaterol therapy in symptomatic chronic obstructive pulmonary disease: a randomized study. Adv Ther. 2017;34(11):2518-33.
11. European Medicines Agency. Guideline on clinical investigation of medicinal products in the treatment of chronic obstructive pulmonary disease (COPD), 2012. Accessed March 1, 2021. https://www.ema.europa.eu/ en/documents/scientific-guideline/ guideline-clinical-investigation-medicinal-products-treatment-chronic-obstructive-pulmonary-disease_en.pdf

12. Moretz C, Sharpsten L, Bengtson LG, et al. Real-world effectiveness of umeclidinium/vilanterol versus fluticasone propionate/salmeterol as initial maintenance therapy for chronic obstructive pulmonary disease (COPD): a retrospective cohort study. Int J Chron Obstruct Pulmon Dis. 2019;14:1721-37.

13. Sharafkhaneh A, Altan AE, Colice GL, et al. A simple rule to identify patients with chronic obstructive pulmonary disease who may need treatment reevaluation. Respir Med. 2014;108(9):1310-20.

14. Charlson ME, Pompei P, Ales KL, MacKenzie CR. A new method of classifying prognostic comorbidity in longitudinal studies: development and validation. J Chronic Dis. 1987;40(5):373-83.

15. Elixhauser A, Steiner C, Harris DR, Coffey RM. Comorbidity measures for use with administrative data. Med Care. 1998;36(1):8-27.

16. Wu EQ, Birnbaum HG, Cifaldi M, Kang Y, Mallet D, Colice G. Development of a COPD severity score. Curr Med Res Opin. 2006;22(9):1679-87.

17. U.S. Bureau of Labor Statistics. Consumer Price Index. Medical care. Series ID: CUUR0000SAM. Accessed March 1, 2021. http://data.bls.gov/ cgi-bin/surveymost?cu

18. Wolter KM. Taylor series methods. Introduction to Variance Estimation. New York: Springer. 1985;221-47.

19. Rosenbaum PR, Rubin DB. The central role of the propensity score in observational studies for causal effects Biometrika. 1983;70(1):41-55. 
20. Stuart EA, Lee BK, Leacy FP. Prognostic score-based balance measures can be a useful diagnostic for propensity score methods in comparative effectiveness research. J Clin Epidemiol. 2013;66(8 Suppl):S84-S90 e81.

21. Andrello AC, Donaria L, de Castro LA, et al. Maximum voluntary ventilation and its relationship with clinical outcomes in subjects with COPD. Respir Care. 2021;66(1):79-86.

22. Palli SR, Buikema AR, DuCharme M, Frazer M, Kaila S, Juday T. Costs, exacerbations and pneumonia after initiating combination tiotropium olodaterol versus triple therapy for chronic obstructive pulmonary disease. J Comp Eff Res. 2019;8(15):1299-316.
23. Palli SR, Frazer M, DuCharme M, Buikema AR, Anderson AJ, Franchino-Elder J. Differences in real-world health and economic outcomes among patients with COPD treated with combination tiotropium/olodaterol versus triple therapy. J Manag Care Spec Pharm. 2020;26(10):1363-74. https://www.jmcp. org/doi/pdf/10.18553/jmcp.2020.20159

24. HCUPnet, Healthcare Cost and Utilization Project. Agency for Healthcare Research and Quality, Rockville, MD. Accessed March 1, 2021. https://hcupnet. ahrq.gov/

25. Dyrda L. 25 facts and statistics on emergency departments in the US. Accessed March 1, 2021. https:// www.beckershospitalreview.com/ hospital-management-administration/25facts-and-statistics-on-emergencydepartments-in-the-us.html
26. Groeger LG. ER inspector: find and evaluate every emergency room near you. ProPublica. Accessed March 1, 2021. https://projects.propublica.org/ emergency

27. Reese P. As ER wait times grow, more patients leave against medical advice. Kaiser Health News. Accessed March 1, 2021. https://khn.org/news/ as-er-wait-times-grow-more-patientsleave-against-medical-advice/

28. UnitedHealthGroup. The high cost of avoidable hospital emergency department visits. Accessed March 1, 2021. https:// www.unitedhealthgroup.com/avoidableed-visits.html

29. Austin PC. An introduction to propensity score methods for reducing the effects of confounding in observational studies. Multivariate Behav Res. 2011;46(3):399-424. 